\title{
Structural assessment of heritage buildings
}

\author{
M. Holicky \& M. Sykora \\ Klokner Institute, Czech Technical University in Prague, Czech Republic
}

\begin{abstract}
Reliability assessment of heritage buildings is in many aspects different from that taken in designing the structure of a newly proposed building. The effects of the construction process and subsequent life of the architecture, during which it may have undergone alteration, deterioration, misuse, and other changes to its as-built (as-designed) state, must be taken into account. That is why the assessment of heritage architecture often requires application of sophisticated methods, as a rule beyond the scope of traditional design practice and codes. The two main principles for the assessment of heritage architecture may be summarized as follows: (1) available scientific knowledge and know-how including currently valid codes should be applied; historical practice and provisions valid when the architecture was built (designed), should be used as guidance information only; (2) actual characteristics of structural material, action, geometric data and structural behaviour should be considered; the original documentation including drawings should be used as guidance material only. The most important step of the whole assessment procedure of heritage architecture is evaluation of inspection data and updating of prior information concerning strength and structural reliability. Typically, assessment of heritage architecture is a cyclic process in which the first preliminary assessment is often supplemented by subsequent detailed investigations and assessment. Keywords: structures, assessment, heritage buildings.
\end{abstract}

\section{Introduction}

Assessment of existing structures is becoming a more and more important and frequent engineering task. Continued use of existing structures is of a great significance due to environmental, economic and socio-political assets, growing larger every year. These aspects are particularly relevant to heritage buildings that always constitute a great historical, social and economic value. 
General principles of sustainable development regularly lead to the need for extension of the life of a structure, in majority of practical cases in conjunction with severe economic constraints. That is why assessment of existing structures often requires application of sophisticated methods, as a rule beyond the scope of traditional design codes. Nevertheless, apart from few national codes, three International Standards ISO 2394 [1], ISO 13822 [2] and ISO 12491 [3], related to assessment of existing structures, have been recently developed. ISO 13822 [2] contains an annex focused on heritage structures. Additional information may be found in a number of scientific papers and publications, see e.g. Melchers [4] and Ellingwood [5].

The approach to assessment of a heritage building is in many aspects different from that taken in designing the structure of a newly proposed building. The effects of the construction process and subsequent life of the structure, during which it may have undergone alteration, deterioration, misuse, and other changes to its as-built (as-designed) state, must be taken into account.

However, even though the heritage building may be investigated several times, some uncertainty in the basic variables and structural behaviour shall always remain. Therefore, similarly as in design of new structures, actual variation in the basic variables describing actions, material properties, geometric data and model uncertainties are taken into account by partial factors or other code provisions.

In general, a heritage structure may be subjected to the assessment of its actual reliability in case of:

- rehabilitation during which new structural members are added to the existing load-carrying system;

- adequacy checking in order to establish whether the heritage structure can resist loads associated with the anticipated change in use of the facility, operational changes or extension of its design working life;

- repair of a heritage building, which has deteriorated due to time dependent environmental effects or which has suffered damage from accidental actions, for example, earthquake;

- doubts concerning actual reliability of the structure.

In some circumstances assessments may also be required by authorities, insurance companies or owners or may be demanded by a maintenance plan.

\section{Principles and general framework of assessment}

Two main principles are usually accepted when assessing heritage buildings:

- Currently valid codes for verification of structural reliability should be applied; historic codes valid in the period when the structure was designed should be used only as guidance documents.

- Actual characteristics of structural materials, actions, geometric data and structural behaviour should be considered, the original design documentation including drawings should be used as guidance only.

The first principle should be applied in order to achieve similar reliability level as in case of newly designed structures. The second principle should avoid 
negligence of any structural condition that may affect actual reliability (in favourable or unfavourable way) of a given structure.

Most of the current codes are developed assuming the concept of limit states in conjunction with the partial factor method. In accordance with this method, which is mostly considered here, basic variables are specified by characteristic or representative values. The design values of basic variables are determined on the basis of the characteristic (representative) values and appropriate partial factors.

It follows from the second principle that a visual inspection of the assessed structure should be made whenever possible. Practical experience shows that inspection of the site is also useful to obtain a good feel for actual situation and state of the structure.

As a rule the assessment need not to be performed for those parts of the structure that will not be affected by structural changes, rehabilitation, repair, change in use or which are not obviously damaged or are not suspected of having insufficient reliability, ISO 13822 [2].

In general, the assessment procedure consists of the following steps (see the flow chart in ISO 13822 [2]):

- specification of the assessment objectives required by the client or authority;

- scenarios related to structural conditions and actions;

- preliminary assessment;

- study of available documentation;

- preliminary inspection;

- preliminary checks;

- decision on immediate actions;

- recommendation for detailed assessment;

- detailed assessment;

- detailed documentary search;

- detailed inspection;

- material testing and determination of actions;

- determination of structural properties;

- structural analysis;

- verification of structural reliability;

- report including proposal for construction intervention;

- repeat the sequence if necessary.

When the preliminary assessment indicates that the structure is reliable for its intended use over the remaining life a detailed assessment may not be required. Conversely if the structure seems to be in dangerous or uncertain condition immediate interventions and detailed assessment may be necessary.

\section{Investigation}

Investigation of a heritage structure is intended to verify and update the knowledge about the present condition (state) of a structure with respect to a number of aspects. Often, the first impression of the structural condition will be 
based on visual qualitative investigation. The description of possible damage of the structure may be presented in verbal terms like: 'unknown, none, minor, moderate, severe, destructive'. Very often the decision based on such an observation will be made by experts in a purely intuitive way.

A better judgement of the structural condition can be made on the basis of (subsequent) quantitative inspections. Typically, the assessment is a cyclic process when the first inspection is supplemented by subsequent investigations. The purpose of the subsequent investigations is to obtain a better feel for the actual structural condition (particularly in the case of damage) and to verify information required for determination of the characteristic and representative values of all basic variables. For all inspection techniques, information on the probability of detecting damages if present, and the accuracy of the results should be given.

The statement from the investigation contains, as a rule, the following data describing

- actual state of the structure;

- types of structural materials and soils;

- observed damages;

- actions including environmental effects;

- available design documentation.

Proof loading is a special type of investigation. Based on such tests one may draw conclusions with respect to:

- the bearing capacity of the tested member under the test load condition;

- other members;

- other load conditions;

- the behaviour of the system.

The inference in the first case is relatively easy; the probability density function of the load bearing capacity is simply cut off at the value of the proof load. The inference from the other conclusions is more complex. Note that the number of proof load tests needs not to be restricted to one. Proof testing may concern one element under various loading conditions and/or a sample of structural elements. In order to avoid an unnecessary damage to the structure due to the proof load, it is recommended to increase the load gradually and to measure the deformations. Measurements may also give a better insight into the behaviour of the system. In general proof loads can address long-term or timedependent effects. These effects should be compensated by calculation.

\section{Basic variables}

In accordance with the above-mentioned general principles, characteristic and representative values of all basic variables shall be determined taking into account the actual situation and state of the structure. Available design documentation is used as a guidance material only. Actual state of the structure should be verified by its inspection to an adequate extent. If appropriate, 
destructive or non-destructive inspections should be performed and evaluated using statistical methods.

For verification of the structural reliability using the partial factor method, the characteristic and representative values of basic variables shall be considered as follows:

(a) Dimensions of the structural elements shall be determined on the basis of adequate measurements. However, when the original design documentation is available and no changes in dimensions have taken place, the nominal dimensions given in the documentation may be used in the analysis.

(b) Load characteristics shall be introduced with the values corresponding with the actual situation verified by destructive or non-destructive inspections. When some loads have been reduced or removed completely, the representative values can be reduced or appropriate partial factors can be adjusted. When overloading has been observed in the past it may be appropriate to increase adequately representative values.

(c) Material properties shall be considered according to the actual state of the structure verified by destructive or non-destructive inspections. When the original design documentation is available and no serious deterioration, design errors or construction errors are suspected, the characteristic values given in original design may be used.

(d) Model uncertainties shall be considered in the same way as in design stage unless previous structural behaviour (especially damage) indicates otherwise. In some cases model factors, coefficients and other design assumptions may be established from measurements on the heritage structure (e.g. wind pressure coefficient, effective width values, etc.).

Thus the reliability verification should be backed up by inspection of the structure including collection of appropriate data. Evaluation of prior information and its updating using newly obtained measurements is one of the most important steps of the assessment.

\section{Evaluation of inspection results}

Using results of an investigation (qualitative inspection, calculations, quantitative inspection, proof loading) the properties and reliability estimates of the structure may be updated. Two different procedures can be distinguished:

(1) Updating of the structural failure probability.

(2) Updating of the probability distributions of basic variables.

Direct updating of the structural reliability (procedure (1)) can be formally carried out using the following basic formula of the probability theory:

$$
\mathrm{P}(F \mid I)=\frac{\mathrm{P}(F \cap I)}{\mathrm{P}(I)}
$$


where $\mathrm{P}$ denotes probability, $F$ local or global failure, $I$ inspection information, and $\cap$ intersection of two events. The inspection information $I$ may consist of the observation that the crack width at the beam B is smaller than at the beam A. An example of probability updating using equation (1) is provided by Sykora et al. [6].

The updating procedure of a univariate or multivariate probability distribution (procedure (2)) is given formally as:

$$
\mathrm{f}_{X}(x \mid I)=C \mathrm{P}(I \mid x) \mathrm{f}_{X}(x)
$$

where $\mathrm{f}_{X}(x \mid I)$ denotes the updated probability density function of $X, \mathrm{f}_{X}(x)$ denotes the probability density function of $X$ before updating, $X$ a basic variable or statistical parameter, $I$ inspection information, $C$ normalising constant, and $\mathrm{P}(I \mid x)$ likelihood function.

An illustration of equation (2) is presented in Figure 1. In this example updating leads to a more favourable distribution with a greater design value $x_{\mathrm{d}}$ than the prior design value $x_{\mathrm{d}}$. In general, however, the updated distribution might be also less favourable than the prior distribution.

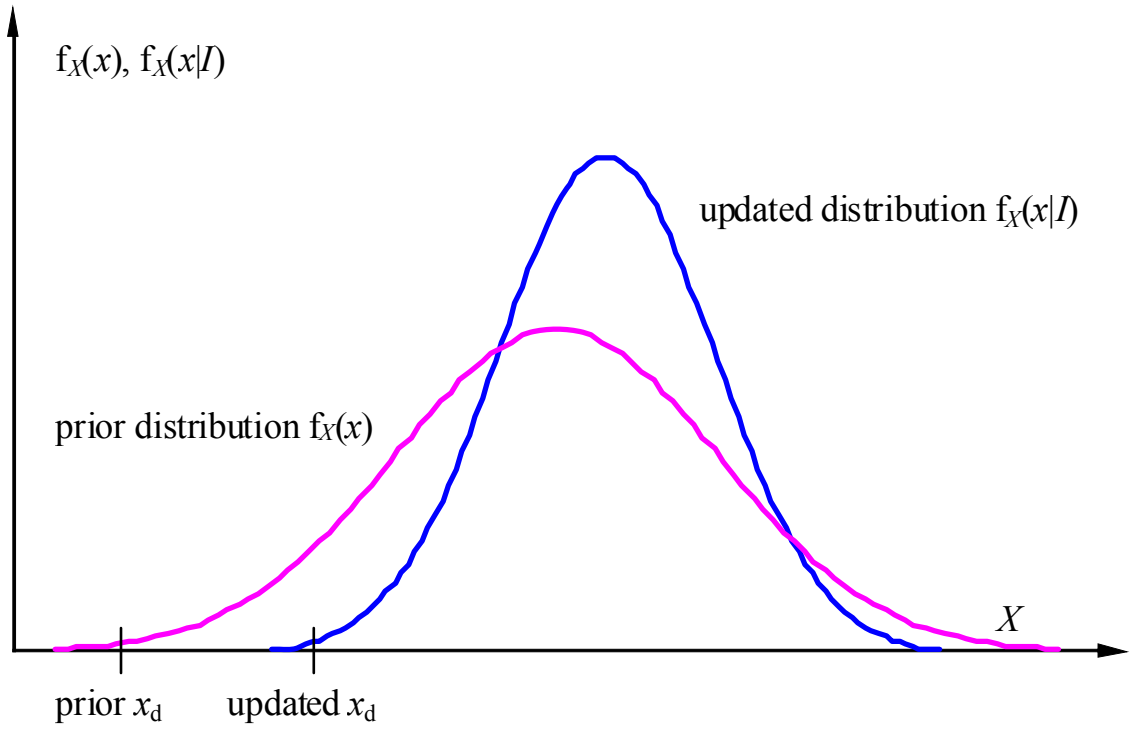

Figure 1: Updating of probability density function for an expected variable $X$.

The updating procedure can be used to derive updated characteristic and representative values (fractiles of appropriate distributions) of basic variables to be used in the partial factor method or to compare directly action effects with limit values (cracks, displacements). More information on updating may be found in ISO 12491 [3].

Once the updated distributions for the basic variables $\mathrm{f}_{X}(x)$ have been found, the updated failure probability $\mathrm{P}(F \mid I)$ may be determined by performing a 
probabilistic analysis using common method of structural reliability for new structures. Symbolically it can be written

$$
\mathrm{P}(F \mid I)=\int_{\mathrm{G}(X)<0} \mathrm{f}_{X}(x \mid I) \mathrm{d} x
$$

where $\mathrm{f}_{X}(x \mid I)$ denotes the updated probability density function and $\mathrm{g}(x)<0$ denotes the failure domain $(\mathrm{g}(x)$ being the limit state function). It should be proved that the probability $\mathrm{P}(F \mid I)$, given the design values for its basic variables, does not exceed a specified target value.

A more practical procedure is to determine updated design values for each basic variable (procedure (2)). For a resistance parameter $X$, the design value can be obtained using operational formula of ISO 2394 [1]. For normal and lognormal random variable it holds, respectively

$$
\begin{gathered}
x_{\mathrm{d}}=\mu(1-\alpha \beta V) \\
x_{\mathrm{d}}=\mu \exp \left(-\alpha \beta \sigma-0.5 \sigma^{2}\right)
\end{gathered}
$$

where $x_{\mathrm{d}}$ is the updated design value for $X, \mu$ updated mean value, $\alpha$ probabilistic influence coefficient, $\beta$ target reliability index, $V$ updated coefficient of variation, and $\sigma^{2}=\ln \left(1+V^{2}\right)$.

The value of the target reliability index $\beta$ is discussed in ISO 13822 [2], the values of $\alpha$ can be taken equal to those commonly used for new structures $(0.7$ for the dominating load parameter, 0.8 for the dominating resistance parameter and 0.3 for non-dominating variables according to ISO 2394 [1]).

As an alternative to procedure (2), one might also determine the characteristic value $x_{\mathrm{k}}$ first and calculate the design value $x_{\mathrm{d}}$ by applying the appropriate partial factor $\gamma_{\mathrm{m}}$

$$
x_{\mathrm{d}}=x_{\mathrm{k}} / \gamma_{\mathrm{m}}
$$

For normal and lognormal random variable $X$ the characteristic value $x_{\mathrm{k}}$ then follows as

$$
\begin{gathered}
(\mathrm{C} .4 \mathrm{a}) \\
x_{\mathrm{k}}=\mu(1-k V) \\
x_{\mathrm{k}}=\mu \exp \left(-k \sigma-0.5 \sigma^{2}\right)
\end{gathered}
$$

where $k=1.64$ (5\% fractile of the standardised normal distribution) is usually used. It may be helpful to consider both methods and to use a more conservative result. This procedure may be applied for all basic variables. However, for geomechanical properties and variable loads usually other distributions apart from the normal and lognormal distribution may be more suitable.

Note that a lower acceptable reliability level can be specified by reducing $\beta$ values for probabilistic design and reducing $\gamma$ - values in the partial factor method. For a material property $X$ described by a normal distribution the partial factor $\gamma_{\mathrm{m}}$ may be estimated using equation

$$
\gamma_{\mathrm{m}}=\frac{x_{k}}{x_{d}}=\frac{\mu-k \sigma}{\mu-\alpha \beta \sigma}
$$


which follows from general relationship (4). All the symbols used in (8) are defined above ( $k=1.64$ is usually used for the characteristic strength). Similar relationships between $\gamma_{\mathrm{m}}$ and $\beta$ may be derived for lognormal or other distributions.

\section{Structural analysis}

Structural behaviour should be analysed using models that describe actual situation and state of a heritage structure. Generally the structure should be analysed for ultimate limit states and serviceability limit states using basic variables and taking into account relevant deterioration processes.

All basic variables describing actions, material properties, load and model uncertainties should be considered as mentioned above. The uncertainty associated with the validity and accuracy of the models should be considered during assessment, either by adopting appropriate factors in deterministic verifications or by introducing probabilistic model factors in reliability analysis.

When a structure is analysed, conversion factors reflecting the influence of shape and size effect of specimens, temperature, moisture, duration-of-load effect, etc., should be taken into account. The level of knowledge about the condition of components should be also considered. This can be achieved by adjusting the assumed variability in either the load carrying capacity of the components or the dimensions of their cross sections, depending on the type of structure.

When deterioration is observed, the relevant mechanisms shall be identified and a deterioration model predicting the future performance of the structure shall be determined on the basis of theoretical or experimental investigation, inspection, and experience.

\section{Verification}

Reliability verification of a heritage building shall be made using valid codes of practice, as a rule based on the limit state concept. Attention should be paid to both the ultimate and serviceability limit states. Verification may be carried out using partial safety factor or structural reliability methods with consideration of structural system and ductility of components. The reliability assessment shall be made taking into account the remaining working life of a structure, the reference period, and changes in the environment of a structure associated with an anticipated change in use.

The conclusion from the assessment shall withstand a plausibility check. In particular, discrepancies between the results of structural analysis (e.g. insufficient safety) and the real structural condition (e.g. no sign of distress or failure, satisfactory structural performance) must be explained. It should be kept in mind that many engineering models are conservative and cannot be always used directly to explain an actual situation.

The target reliability level used for verification can be taken as the level of reliability implied by acceptance criteria defined in proved and accepted design 
codes. The target reliability level shall be stated together with clearly defined limit state functions and specific models of the basic variables.

The target reliability level can also be established taking into account the required performance level for the structure, the reference period and possible failure consequences. In accordance with ISO 2394 [1], the performance requirements for assessment of existing structures are the same as for design of a new structure. Lower reliability targets for existing structures may be used if they can be justified on the basis of economical, social and sustainable consideration (see Annex F to ISO 13822 [2] and numerical example provided by Sykora et al. [7]).

An adequate value of the reliability index $\beta$ should be in general determined considering appropriate reference period, ISO 13822 [2]. For serviceability and fatigue the reference period equals the remaining working life, while for the ultimate limit states the reference period is in principle the same as the design working life specified for new structures (50 years for buildings). This general approach should be in specific cases supplemented by detailed consideration of the character of serviceability limit states (reversible, irreversible); fatigue (controllable, incontrollable) and consequences of ultimate limit states (loss of the cultural heritage value, economic consequences, number of endangered people).

\section{Assessment in the case of damage}

For an assessment of a damaged structure the following stepwise procedure is recommended:

1) Visual inspection

It is always useful to make an initial visual inspection of the structure to get a feel for its condition. Major defects should be reasonably evident to the experienced eye. In the case of very severe damage, immediate measures (like abandonment of the structure) may be taken.

2) Explanation of observed phenomena

To be able to understand the present condition of the structure, one should simulate the damage or the observed behaviour, using a model of the structure and the estimated intensity of various loads or physical/chemical agencies. It is important to have available documentation with respect to design, analysis and construction. If there is a discrepancy between calculations and observations, it might be worthwhile to look for design errors, errors in construction, etc.

3) Reliability assessment

Given the structure in its present state and given the present information, the reliability of the structure is estimated, either by means of a failure probability or by means of partial factors. The model of the present structure may be different from the original model. If the reliability is sufficient (i.e. better than commonly accepted in design) one might be satisfied and no further action is required. 
4) Additional information

If the reliability according to step 3 is insufficient, one may look for additional information from more advanced structural models, additional inspections and measurements or actual load assessment.

5) Final decision

If the degree of reliability is still too low, one might decide to:

- accept the present situation for economical reasons;

- reduce the load on the structure;

- repair the building;

- start demolition of the structure.

The first decision may be motivated by the fact that the cost for additional reliability is much higher for existing structure than for a new structure. This argument is sometimes used by those who claim that a higher reliability should be generally required for a new structure than for an existing one. However, if human safety is involved, economical optimisation has a limited significance.

\section{Final report and decision}

The final report on structural assessment and possible interim reports (if required) should include clear conclusions with regard to the objective of the assessment based on careful reliability assessment and cost of repair or upgrading. The report shall be concise and clear. A recommended report format is indicated in Annex G to ISO 13822 [2].

If the reliability of a structure is sufficient, no action is required. If an assessment shows that the reliability of a structure is insufficient, appropriate interventions should be proposed. Temporary intervention may be recommended and proposed by the engineer if required immediately. The engineer should indicate a preferred solution as a logical follow-up to the whole assessment in every case.

It should be noted that the client in collaboration with the relevant authority should make the final decision on possible interventions, based on engineering assessment and recommendations. The engineer performing the assessment might have, however, the legal duty to inform the relevant authority if the client does not respond in a reasonable time.

Minimisation of construction interventions is required in rehabilitation and upgrades, but sufficient reliability should also be guaranteed. When dealing with the preservation of heritage buildings, it may be difficult to propose construction interventions that respect all requirements for preservation of the heritage value. Modern principles of interventions seem to include the following aspects:

- Unobtrusiveness and respect of the original conception,

- Safety of the construction,

- Durability of materials,

- Balance between costs and available financial resources, and, in some cases also:

- Removability,

- Compatibility of materials, 
- Indoor environment quality including aspects of comfort, security and accessibility.

\section{Concluding remarks}

The main principles for assessment of heritage structures are:

- Currently valid codes for verification of structural reliability should be applied, historic codes valid in the period when the structure was designed, should be used only as guidance documents;

- Actual characteristics of structural material, action, geometric data and structural behaviour should be considered; original design documentation including drawings should be used as guidance material only.

The most important step of the whole assessment procedure is evaluation of inspection data and updating of prior information concerning strength and structural reliability. It appears that a Bayesian approach can provide an effective tool.

Typically, assessment of the heritage structures is a cyclic process in which the first preliminary assessment is often supplemented by subsequent detailed investigations and assessment. A report on structural assessment prepared by an engineer should include a recommendation on possible intervention. However, the client in collaboration with the relevant authority should make the final decision concerning possible interventions.

\section{Acknowledgement}

This study is an outcome of the research project DF12P01OVV040 "Assessment of safety and working life of industrial heritage buildings".

\section{References}

[1] ISO 2394 (1998) General principles on reliability of structures. ISO, Geneva, Switzerland.

[2] ISO 13822 (2001) Basis for design of structures -Assessment of existing structures. ISO, Geneva, Switzerland.

[3] ISO 12491 (1998) Statistical methods for quality control of building materials and components. ISO, Geneva, Switzerland.

[4] Melchers R.E. (2001) Structural reliability analysis and prediction. John Wiley and Sons.

[5] Ellingwood B.R. (1996) Reliability-based condition assessment and LRFD for existing structures. Structural Safety, 18 (2+3), 67-80.

[6] Sykora M., Holicky M. and Markova J. (2010) Advanced assessment of industrial heritage buildings for sustainable cities' development. In 
80 Defence Sites

Proceedings of CESB 10, Prague, 30 June - 2 July 2010. Prague: Grada Publishing, p.9.

[7] Sýkora M. et al. (2010) Optimum Target Reliability Levels for Industrial Heritage Structures. In Proceedings of ESREL 2010, Rhodes, Greece, 5 - 9 September 2010. Leiden: Balkema/CRC Press, p.1519-1526. 